\title{
Surface Roughness and Porosity of Hydrated Cement Pastes
}

\author{
T. Ficker, D. Martišek, H. M. Jennings
}

\begin{abstract}
3D profile and roughness parameters were used to perform an extensive study of the fracture surfaces of hydrated cement pastes. One hundred and eight specimens were prepared with six different water-to-cement ratios. The surfaces of the fractured specimens are the subject of a study analysing the height irregularities and the roughness. The values of the 3D surface profile parameters and the roughness parameters of the fractured specimens were computed from digital replicas of surface reliefs assembled by means of confocal microscopy in three different magnifications: $5 \times, 20 \times$ and $50 \times$. Seventy-eight graphs were plotted to describe and analyze the dependences of the height and roughness irregularities on the water-to-cement ratio and on the porosity of the cement hydrates. The results showed unambiguously that the water-to-cement ratio or equivalently the porosity of the specimens has a decisive influence on the irregularities of the fracture surfaces of this material. The experimental results indicated the possibility that the porosity or the value of the water-to-cement ratio might be inferred from the height irregularities of the fracture surfaces. It was hypothesized that there may be a similarly strong correlation between porosity and surface irregularity, on the one hand, and some other highly porous solids, on the other, and thus the same possibility to infer porosity from the surfaces of their fracture remnants.
\end{abstract}

Keywords: roughness analysis, fracture surfaces, cement-based materials, confocal microscopy.

\section{Introduction}

The surface features of fractured materials are valuable sources of information on the topological, structural and mechanical properties of the materials. However, in the research field of cementitious materials there have only been a restricted number of studies dealing with the surface features of fractured specimens. Some early surface studies of hydrated cement materials focused on fractal properties $[1,2]$, whereas others [3-5] investigated roughness numbers $(R N)$ or similar surface characteristics $[6-8]$.

In the $R N$ studies [3-5] dealing with fracture surfaces, the authors used as a research tool the so-called roughness numbers $R N$, which proved to be correlated to energy-based mechanical quantities e.g. toughness but, unfortunately, they did not show a correlation with porosity-dependent quantities, e.g. compressive strength. In our preliminary study [9], it was illustrated that, in addition to the roughness numbers $R N$, there are very promising surface parameters of another kind called the surface profile $(S P)$ and surface roughness $(S R)$ parameters derived from three-dimensional (3D) digital replicas of fracture surfaces. It has been indicated [9] that these $S P$ and $S R$ parameters, in contrast to $R N$, might show a correlation with porosity and with porosity-based quantities such as compressive strength and, as such, they could become an important complement to $R N$ characteristics.
As has been highlighted previously [9], the striking difference between $R N$ and $S P / S R$ characteristics consists in their definitions. $R N$ numbers quantify the increase in the area of fracture surfaces, i.e. they are overall area characteristics, whereas $S P / S R$ parameters evaluate surface irregularities, i.e. they quantify height differences on fracture reliefs.

In our preliminary report [9], only three types of $S P / S R$ parameters were tested, though there is a larger series of these $3 \mathrm{D}$ parameters. In addition, in the preliminary report [9] the tested specimens covered only four values of the water-to-cement ratio $r$, namely $0.4,0.6,0.8$, and 1.0 , though the reliability of the experimental conclusion is increased by including more values of $r$. Last but not least, some vagueness may be added to the results by the porosity of specimens inferred from the r-values that are used, rather than from direct measurements. All these points have been improved in the present communication, which is based on a larger series of $S P / S R$ parameters, on a more numerous spectrum of $r$-values, on direct measurements of porosity and, in addition, on a larger number of specimens. This makes the present results more reliable and more precise than the preliminary results [9].

The goal of the present communication is to provide clear experimental evidence showing porosity as a main factor controlling the irregularity of the fracture surfaces of porous materials. For this purpose, the behavior of the profile parameters $S P$ and roughness parameters $S R$ has been analyzed in terms of various porosity values. 


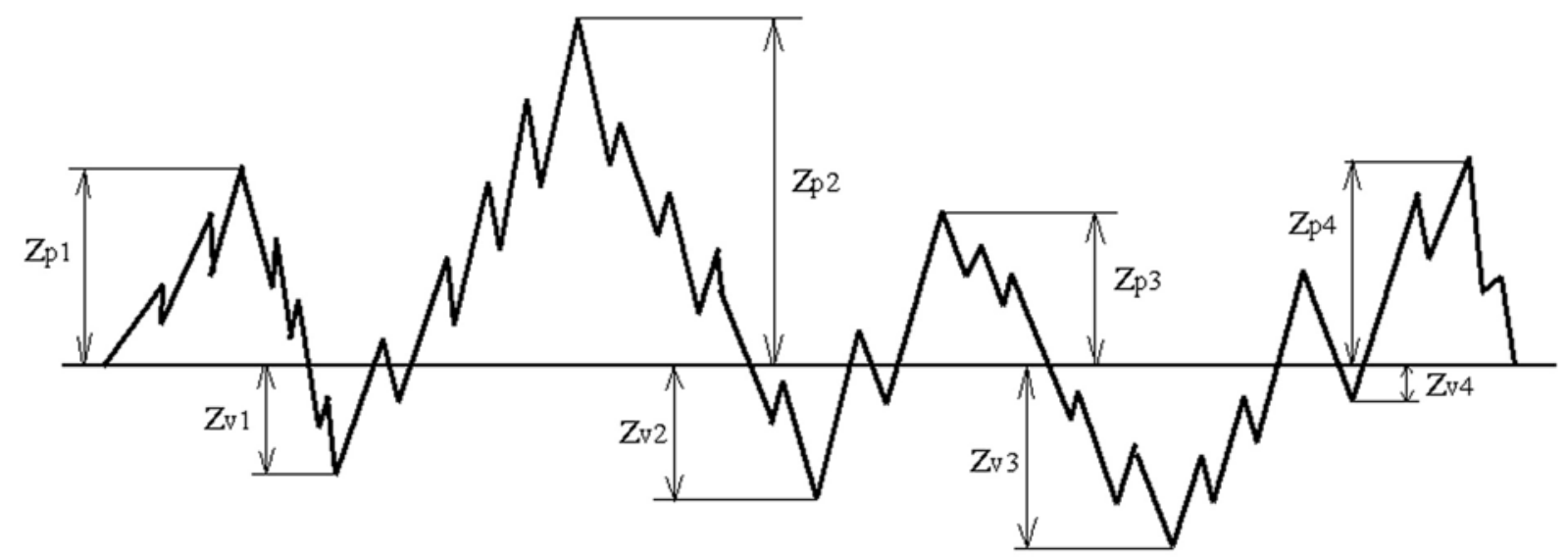

Fig. 1: Scheme of surface irregularities

\section{Surface profile and roughness parameters}

In this study, six different profile parameters $\left(S P_{p}\right.$, $\left.S P_{v}, S P_{z}, S P_{c}, S P_{a}, S P_{q},\right)$ and seven different roughness parameters $\left(S R_{p}, S R_{v}, S R_{z}, S R_{c}, S R_{a}, S R_{q}\right.$, $\left.S R_{z j i s}\right)$ are employed. This means that thirteen independent parameters are used as a measure of the irregularity of fracture surfaces. All these parameters are derived from 3D digital replicas (maps) of the surfaces. In our case, the digital maps have been created by means of confocal microscopy (for more details, see [9]). The map may be viewed as a threedimensional matrix $\left[x_{i}, y_{i}, z_{i}\right]$ where the discrete function $z_{i}=f\left(x_{i}, y_{i}\right)$ approximates the fracture surface, which is in reality more or less a smooth wavy surface $z=f(x, y)$.

$S P$ parameters are derived from the measured map $f\left(x_{i}, y_{i}\right)$, whereas $S R$ parameters are associated with a different function $f_{R}\left(x_{i}, y_{i}\right)$ formed from $f\left(x_{i}, y_{i}\right)$ by filtering out the wavy components possessing wave lengths longer than a critical value $\lambda_{c}$ (for more details see [9]). Briefly, $S P$ parameters characterize surfaces on all measured length scales, but $S R$ chracterizes those length scales that are shorter than $\lambda_{c}$. The algorithms for the computation of $S P$ and $S R$ parameters are very similar; only their source functions differ. To determine $S P$ the starting function is given by $f\left(x_{i}, y_{i}\right)$, whereas for $S R$ it is the filtered function $f_{R}\left(x_{i}, y_{i}\right)$. The computational algorithms are briefly explained in the following paragraphs with the aid of Figure 1, which has been drawn for simplicity in two dimensions rather than in three dimensions.

Parameters $S P_{p} / S R_{p}$ - these parameters represent the total maxima on the graphs of the source functions $f\left(x_{i}, y_{i}\right) / f_{R}\left(x_{i}, y_{i}\right)$ (Figure 1), i.e.

$$
S P_{p} / S R_{p} \rightarrow \text { total } \max \left\{z_{p i}\right\}
$$

Parameters $S P_{v} / S R_{v}$ - inform us about the total minima on the graphs of the source functions $f\left(x_{i}, y_{i}\right) / f_{R}\left(x_{i}, y_{i}\right)$ (Figure 1), i.e.

$$
S P_{v} / S R_{v} \rightarrow \text { total } \min \left\{z_{v i}\right\}
$$

Parameters $S P_{z} / S R_{z}$ - are determined by the sum of the total maximum and the total minimum on the graphs of the source functions $f\left(x_{i}, y_{i}\right) / f_{R}\left(x_{i}, y_{i}\right)$ (Figure 1), i.e.

$$
S P_{z} / S R_{z} \rightarrow \text { total } \max \left\{z_{p i}\right\}+\text { total } \min \left\{z_{v i}\right\}
$$

Parameters $S P_{c} / S R_{c}$ - represent the arithmetic averages of the sums of the local maxima and minima on the graphs of the source functions (Figure 1), i.e.

$$
\begin{aligned}
& S P_{c} / S R_{c} \rightarrow \\
& \left.\frac{1}{m} \sum_{i=1}^{m} \text { (local } \max \left\{z_{p i}\right\}+\text { local } \min \left\{z_{v i}\right\}\right)
\end{aligned}
$$

where $m$ is an overall number of local extremes (local maxima and local minima).

Parameters $S P_{a} / S R_{a}$ - are given as mean heights of the graphs of the source functions $f\left(x_{i}, y_{i}\right) / f_{R}\left(x_{i}, y_{i}\right)$ (Figure 1), i.e.

$$
\begin{aligned}
S P_{a} & =\frac{1}{L \cdot M} \iint_{(L M)}|f(x, y)| \mathrm{d} x \mathrm{~d} y \\
S R_{a} & =\frac{1}{L \cdot M} \iint_{(L M)}\left|f_{R}(x, y)\right| \mathrm{d} x \mathrm{~d} y
\end{aligned}
$$

where $L \times M$ is the area of vertical projection of $f(x, y) / f_{R}(x, y)$ into the plane $x y$.

Parameters $S P_{q} / S R_{q}$ - provide us with the square roots of the mean of the squares of the source functions $f\left(x_{i}, y_{i}\right) / f_{R}\left(x_{i}, y_{i}\right)-\mathrm{rms}$ values (Figure 1), i.e.

$$
\begin{aligned}
S P_{q} & =\sqrt{\frac{1}{L \cdot M} \iint_{(L M)}[f(x, y)]^{2} \mathrm{~d} x \mathrm{~d} y} \\
S R_{q} & =\sqrt{\frac{1}{L \cdot M} \iint_{(L M)}\left[f_{R}(x, y)\right]^{2} \mathrm{~d} x \mathrm{~d} y}
\end{aligned}
$$


Parameter $S R_{z j i s}$ - represents the ten-point arithmetic average of the five highest peaks and the five deepest valleys of the source function $f_{R}(x, y)$ (Figure 1), i.e.

$$
S R_{z j i s} \rightarrow \frac{1}{5} \sum_{i=1}^{5}\left(z_{p i}^{\max 5}+z_{v i}^{\min 5}\right)
$$

Analyzing the functionality and the capability of parameters $S P / S R$ defined above, we can recognize their characteristic properties, which make them unique and distinguishable. For example, the first three parameters of both kinds, i.e. $S P_{p}, S P_{v}, S P_{z}$, and $S R_{p}, S R_{v}, S R_{z}$ have only a local character, since each of them determines a value belonging to a single point of the surface (i.e. the local extreme) without taking into account the influence of other surface points. In other words, they select from the very numerous set of peaks, valleys and z-widths only one value, i.e. the extreme value. From the viewpoint of statistics, the representativeness of such values is very limited and may suffer from essential variability. In addition, the $z$-modifications of the $S P$ or $S R$ parameters may be expected partly to resemble the behavior of $p$ - or $v$-modifications of these parameters, since the $z$-modification is actually a combination of $p$ - and $v$-modifications. For all these reasons, the groups of parameters $S P_{p}, S P_{v}, S P_{z}$, and $S R_{p}, S R_{v}$, $S R_{z}$ should be considered as statistically less relevant than the remaining parameters $S P_{c}, S P_{a}, S P_{q}$, or $S R_{c}, S R_{a}, S R_{q}, S R_{z j i s}$, since these two groups represent averaged quantities. Nevertheless, a certain specificity may be observed with parameter $S R_{z j i s}$. Although defined as an averaged quantity, it is not a complete average since the averaging does not go over all the surface features but only over the five highest peaks and the five deepest valleys. Due to this circumstance, $S R_{z j i s}$ also ranks among the statistically less reliable characteristics.

The parameters $S P_{c}, S P_{a}, S P_{q}$, and $S R_{c}, S R_{a}$, $S R_{q}$ represent true averages. Their $c$-components are the results of averaging over all the local $z$-widths, which consist of the $p$ - and $v$-components of the given surface relief, and as such the $c$-components include properties of both the 'upper' and the 'lower' sides of the relief. This makes them rather prone to imitate the behavior of the $p$ - and $v$-components.

During the computation of the $a$ - and $q$ components the entire 'lower halves' of the surface reliefs are 'rotated' up, and in this way they are unified with the 'upper' parts of the reliefs. Together they create a statistically indistinguishable unit which does not suffer so much from the 'twoside effect'. The $a$ - and $q$-components seem to be statistically the most reliable components among all those employed in this study. However, there is a certain difference between $S P_{a} / S P_{q}$ and $S R_{a} / S R_{q}$ pa- rameters. The $R$-parameters are extracted from the roughness function $f_{R}\left(x_{i}, y_{i}\right)$, which lacks the larger wave lengths of the wavy profiles, and this helps $S P_{a}$ and $S P_{q}$ to be better representatives of the overall surface irregularity of fracture specimens.

Finally, it should be mentioned that there are some differences between the surface parameters measured at different magnifications. In the case of smaller magnifications, priority is given to the larger surface features of reliefs but the details are suppressed. This means that the values of all parameters will be shifted to larger quantities - they are set on scales of greater length. At greater magnifications the situation is opposite: the details are accented but the larger relief features are missing, so that the values of all parameters will be shifted to smaller quantities - they are set on scales of shorter length. These properties make all the surface parameters scale-dependent quantities. The surface places (sites) on which measurements were performed are specified in Section 3.

All the mentioned properties of the profile parameters $(S P)$ and roughness parameters $(S R)$ can be observed with the graphs presented in Section 4 .

\section{Experimental arrangement}

One hundred eight specimens $(2 \mathrm{~cm} \times 2 \mathrm{~cm} \times 16 \mathrm{~cm})$ of hydrated ordinary Portland cement paste of six water-to-cement ratios $r(0.3,0.4,0.5,0.6,0.7,0.8)$ were prepared (eighteen samples per $r$-value). The specimens were rotated during hydration to achieve better homogeneity. All specimens were stored for the whole hydration time at $100 \% \mathrm{RH}$ and $20^{\circ} \mathrm{C}$. After 60 days of hydration, the specimens were fractured in three-point bending and the fracture surfaces were immediately used for microscopic analysis. Other parts of the specimens were used for porosity measurements and for further mechanical tests.

Porosity was determined by the common weightvolume method. The wet specimens were weighed and their volume was measured. Then they were subjected to a temperature of $105^{\circ} \mathrm{C}$ for one week until their weight stopped changing, and the dry specimens were weighed again.

The microscopic analysis was performed using an Olympus Lext 3100 confocal microscope. Approximately 150 image sections were taken for each measured surface site, from the very bottom of the surface depressions (valleys) to the very top of the surface protrusions (peaks). The investigated area $L \times M=1280 \mu \mathrm{m} \times 1280 \mu \mathrm{m}(1024$ pixels $\times 1024$ pixels) was chosen in five different places of each fracture surface (in the center, and in four positions near the corners of the rectangular area), i.e. each plotted point in the graphs of the profile and roughness parameters corresponds to an average value composed 


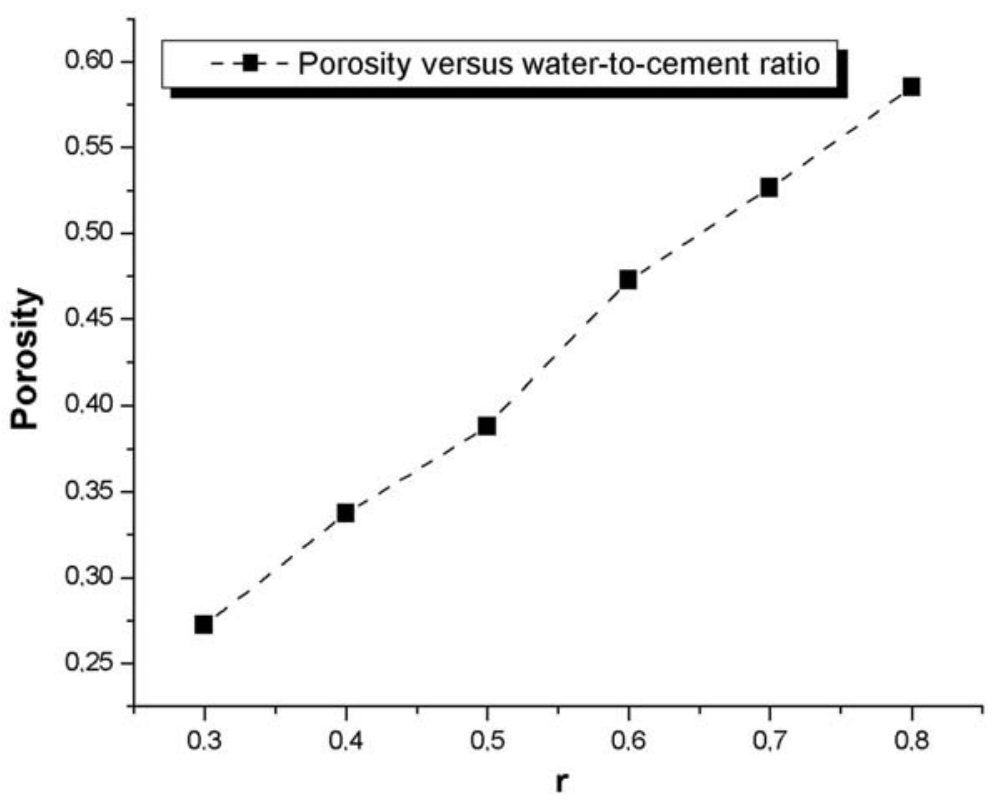

Fig. 2: The dependence of porosity $P$ (measured by evaporable water content) on the original water-to-cement ratio $r$

of 90 measurements (18 samples $\times 5$ surface measurements). Each measurement was performed for three different magnifications, namely $5 \times, 20 \times$ and $50 \times$, giving 270 measurements performed for the particular $r$-value. Each site measurement amounts about 150 optical sections (digital files), i.e. 40500 files had to be processed to create 270 digital maps per one $r$-value. This resulted in 1620 digital maps for all $r$-values altogether $(6 r$-values $\times 270$ maps for each $r$-value). These 1620 digital fracture surfaces were then subjected to $3 \mathrm{D}$ profile and roughness surface analyses using Olympus Lext 3100 software, version 6 . The critical wavelength $\lambda_{c}$ for filtering out wavy components of longer wavelength was set to 100 pixels, which is about $10 \%$ of the reference length $L=1024$ pixels.

In this way an extensive statistical ensemble was created, providing a sufficiently reliable basis for making relevant conclusions.

\section{Results and discussion}

Prior to a discussion of the graphs of profile and roughness parameters, it is necessary to recall some basic facts about the process of forming porosity with hydrated cement pastes.

When cement is mixed with water, the hydration process combines some water into the $\mathrm{C}-\mathrm{S}-\mathrm{H}^{1}$ gel, a main hydration product, and the remaining water is either physically adsorbed in tiny gel pores or remains as free water in the capillary pores. When the water-to-cement ratio $r=w / c$ is increased, the capillary water increases, and along with it the capillary space extends within the hydrated cement paste. The higher the ratio $r$, the larger the capillary space. Naturally, this scheme holds only when all the free water is integrated into the paste. At extremely high ratios $(r>0.6)$, this is a problem because of sedimentation and segregation of cement grains. Nevertheless, rotation of specimens and adding admixtures preserves sufficient homogeneity of the hydrated specimens.

It follows from the foregoing paragraph that the main factor controlling the capillary porosity of cement paste is the water-to-cement ratio, $r$, which is primarily reflected in the total porosity $P$. Therefore, strong dependence of porosity on the r-ratio, i.e. a strong correlation $P(r)$, is expected. This wellknown relationship is not surprising, and it also works with our specimens - see Figure 2. At first sight, graph $P(r)$ in Figure 2 seems to be linear. However, some caution is necessary when inspecting functional behavior within a narrow interval. Many graphs of non-linear functions seem to be almost linear in very narrow intervals. It is necessary to observe the behavior in a wider interval. In our case, the porosity cannot exceed a value of 1 , but it can approach this value for large $r$-ratios, i.e.

$$
\lim _{r \rightarrow \infty} P(r)=1
$$

This requirement cannot be guaranteed by any common linear function, but can be guaranteed by a nonlinear function, e.g. by the exponentially growing function $P(r)=1-\exp \left(-r / r_{o}\right)$ or by some type of a hyperbolically growing function, to mention only some of the possible candidates. Regardless of the type of $P(r)$ function, one fact is clear: $P(r)$ in the interval $r \in(0.3,0.8)$ with our specimens is only

\footnotetext{
${ }^{1} \mathrm{C}-\mathrm{S}-\mathrm{H}$ gel in cement notation: $\mathrm{C}=\mathrm{CaO}, \mathrm{S}=\mathrm{SiO}_{2}, \mathrm{H}=\mathrm{H}_{2} \mathrm{O}$.
} 
slightly non-linear, which qualifies the linear approximation as a possible tentative candidate for $P(r)$ in this interval. As we shall see later, this will have a special impact on the investigated graphical dependences.

Let us briefly summarize the facts that have been formulated in the foregoing lines. The initial value of the water-to-cement ratio $r$ determines the value of the porosity $P$ of hydrated cement paste, which leads to a strongly correlated dependence $P(r)$. This inevitably leads to the conclusion that all the quantities dependent on the water-tocement ratio, $r$, must also be dependent on porosity $P$, and vice versa. In our case these consequences are perfectly fulfilled within the series of 78 graphs in Figures $3-15$. The series of graphs contains six graphs $S P_{p}(r), S P_{v}(r), S P_{z}(r), S P_{c}(r), S P_{a}(r), S P_{q}(r)$, seven graphs $S R_{p}(r), \quad S R_{v}(r), \quad S R_{z}(r), \quad S R_{c}(r)$, $S R_{a}(r), \quad S R_{q}(r), \quad S R_{z j i s}(r)$, six graphs $S P_{p}(P)$, $S P_{v}(P), S P_{z}(P), S P_{c}(P), S P_{a}(P), S P_{q}(P)$, and seven graphs $S R_{p}(P), S R_{v}(P), S R_{z}(P), S R_{c}(P)$, $S R_{a}(P), S R_{q}(P), S R_{z j i s}(P)$. All the graphs are repeated in three different magnifications: $5 \times, 20 \times$ and $50 \times$. The graphs document in a very straightforward manner the strong dependence of both the profile parameters and the roughness parameters on the waterto-cement ratio $r$, and also on porosity $P$. In addition, these dependences on $r$ and $P$ are very similar in shape within the investigated intervals, which is also a consequence of the almost linear behavior of $P(r)$.

All the graphs contain error bars that seem to be rather large. This is because they represent limiting statistical errors, i.e. intervals with $99.73 \%$ confidence. In normal laboratory practice, statistical intervals with $50 \%$ confidence are usually used and as such they would be $4.5 \times$ shorter. However, the limiting intervals are more instructive since they allow us to recognize other possible positions of measured points, and enable us to consider other possible shapes of the graphs.

The next Section discusses the plotted graphs in greater detail.

\subsection{Dependences on the water-to-cement ratio}

Graphs of the dependences $S P_{p}(r), S P_{v}(r), S P_{z}(r)$, $S P_{c}(r), \quad S P_{a}(r), \quad S P_{q}(r)$ and $S R_{p}(r), \quad S R_{v}(r)$, $S R_{z}(r), \quad S R_{c}(r), \quad S R_{a}(r), \quad S R_{q}(r), \quad S R_{z j i s}(r)$ are shown in the upper halves of Figures $3-14$ and in Figure 15. When comparing these graphical results for different magnifications $(5 \times, 20 \times, 50 \times)$, it is obvious that both the $S P$ profile parameters and the $S R$ roughness parameters change their numerical extent according to the magnification. For example, in Figure 3 (magnification $5 \times$ ) the numerical extent of the $S P_{p}$ parameter is $280 \mu \mathrm{m}$, in Figure 4 (magnification $20 \times$ ) the value is $110 \mu \mathrm{m}$, and in Figure 5 (magnification $50 \times$ ) the value is only $62 \mu \mathrm{m}$. This is in full agreement with what was mentioned in Section 2 about the scale-dependent properties of $S P / S R$ parameters.

An inspection of all the $S P / S R$ graphs within the framework of all the magnifications used here, $5 \times$, $20 \times, 50 \times$, results in the conclusion that the smallest statistical scatter of the measured values (not the error bars) can be found in the graphs associated with magnification $20 \times$. It is likely that this magnification is set at the most favorable length scales characteristic for the studied fracture surfaces. At magnification $5 \mathrm{x}$, the fine length scales are not included in the measurements. Thus a larger statistical scatter can be observed at the side of the small water-tocement ratios, where finer fracture surfaces, i.e. finer length scales, are localized (see Figures 3, 6, 9, 12 or 15$)$. On the other hand, at magnification $50 \times$ the coarser length scales of the fracture surfaces are excluded. Thus a larger scatter appears at the side of the higher water-to-cement ratios, since coarser surfaces (with larger length scales) are localized there (see Figures 5, 11 or 15). Intermediate magnification $20 \times$ is optimum for covering the characteristic length scales of the studied surfaces. Thus it shows the smallest statistical scatter of the $S P / S R$ parameter values. Similarly, we can determine some parameters whose behavior is almost unaffected by statistical scatter. Parameters $S P_{a}$ and $S P_{q}$ measured at magnification $20 \times$ show almost smooth behavior, with no major scatter of their values. Parameters $S R_{a}$ and $S R_{q}$ are less representative than parameters $S P_{a}$ and $S P_{q}$ due to their filtered large length scales.

Analyzing the mutual differences between $p$-, $v$-, $z$-components and $c$-, $a$-, $q$-components with both the $S P$ and $S R$ parameters, it is obvious that the larger statistical scatter is most pronounced with the $p$-, $v$-, $z$-components (compare, e.g., Figures 3 and 6). As was highlighted in Section 2, this is because the $p$-, $v$-, $z$-components are not averaged over the fracture surface, while the $c^{-}, a-, q$-components are true averages.

It is interesting to compare the behavior of the $z$-components with the behavior of the $p$-, $v$ components. For example, Figures 5 shows that the $S P_{v}$ parameter records the reduction in surface irregularity (the depth reduction of the deepest valley) at high water-to-cement ratios 0.8 , while the $S P_{p}$ parameter shows no reduction, and $S P_{z}$ - as a combination of the former two parameters - reports a clear reduction in surface irregularity at this point. Naturally, this is a consequence of the definition of the $S P_{z}$ parameter, which consists in the sum of $S P_{p}$ and $S P_{v}$. Moreover, $S P_{z}$ partly influences $S P_{c}$ for similar reasons. In Figures 5 and 8, the drop in sur- 

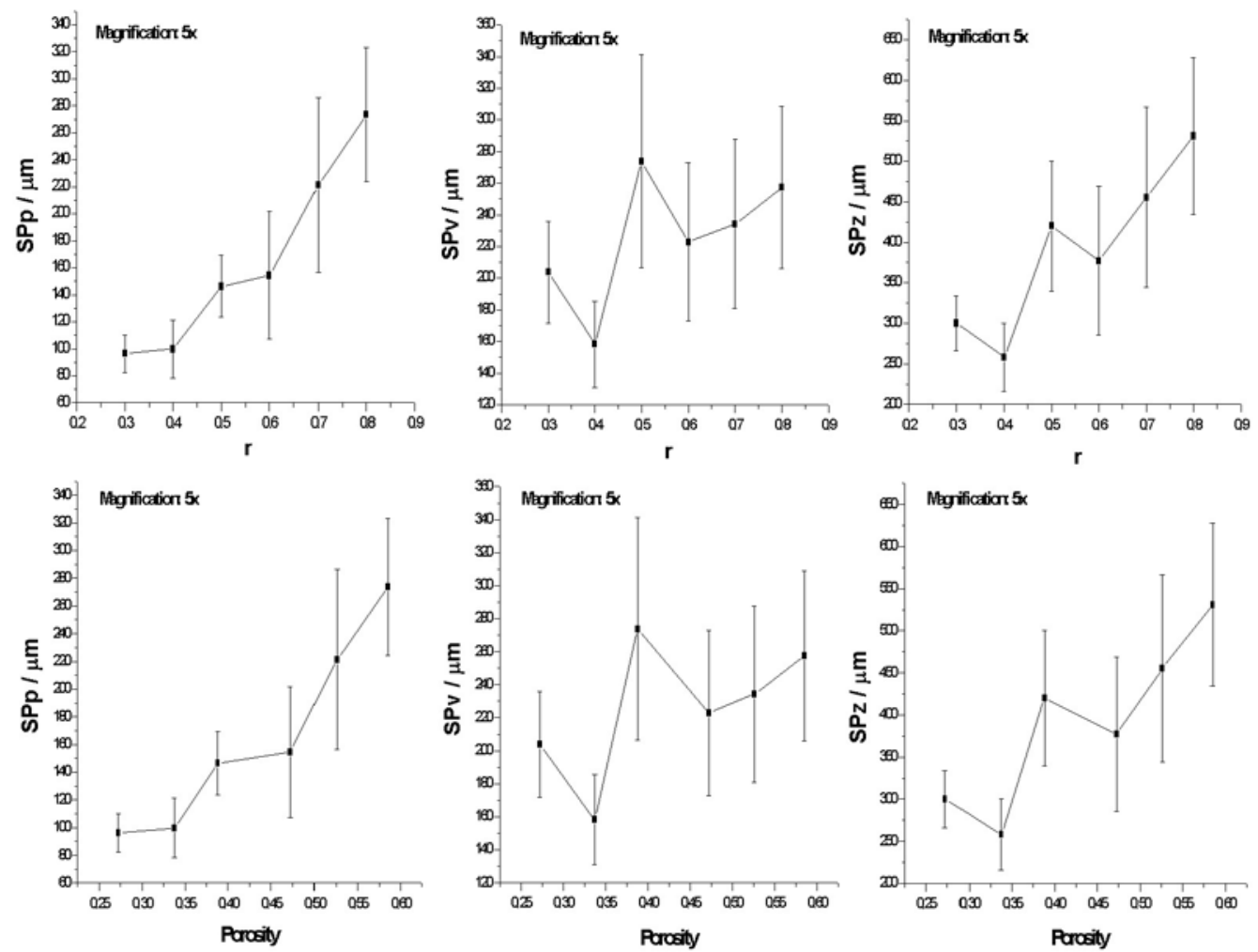

Fig. 3: 3D profile parameters $S P_{p}, S P_{v}, S P_{z}$ as dependents on the water-to-cement ratio $r$ and porosity $P$ - magnification $5 \times$
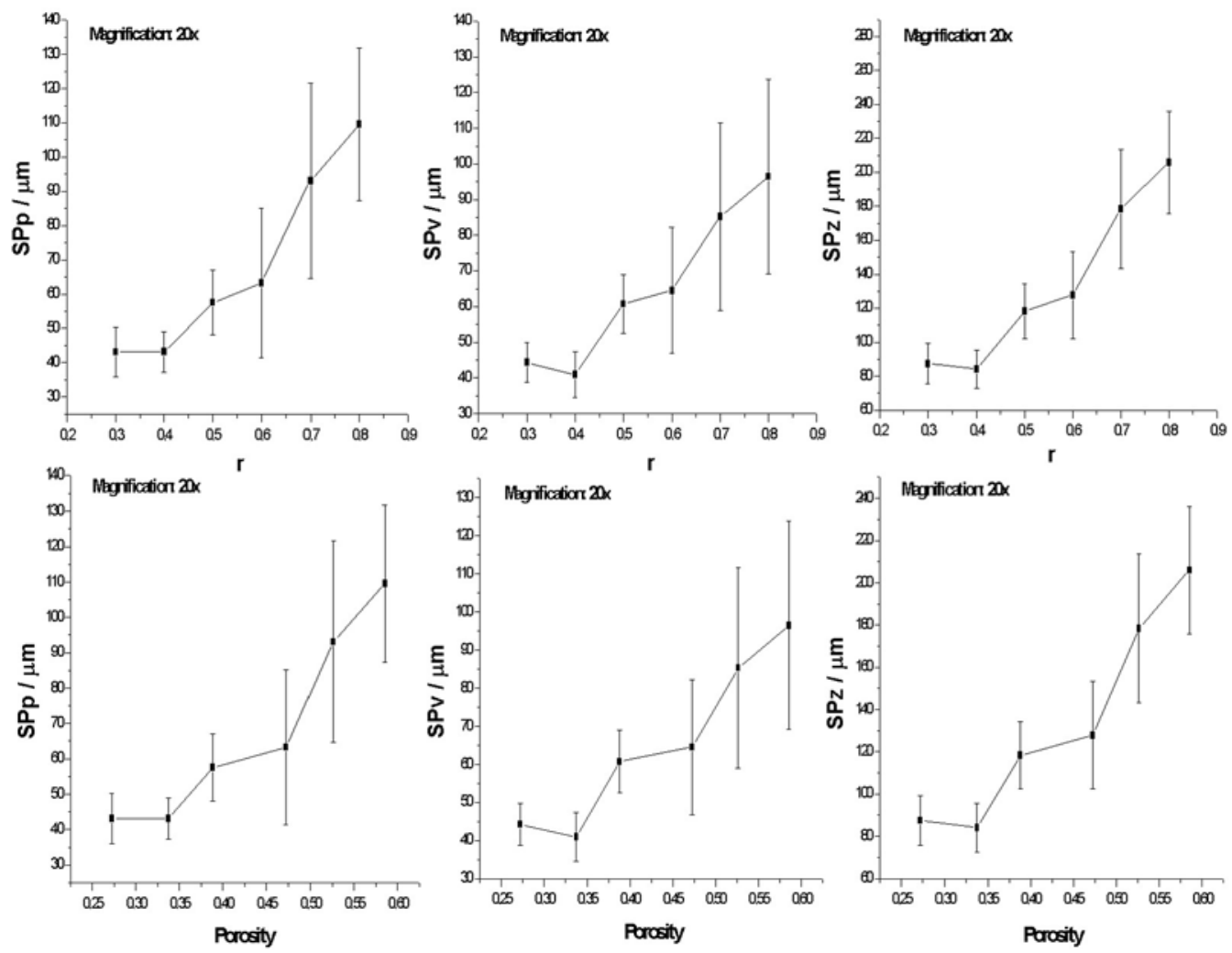

Fig. 4: 3D profile parameters $S P_{p}, S P_{v}, S P_{z}$ as dependents on the water-to-cement ratio $r$ and porosity $P$ - magnification $20 \times$ 

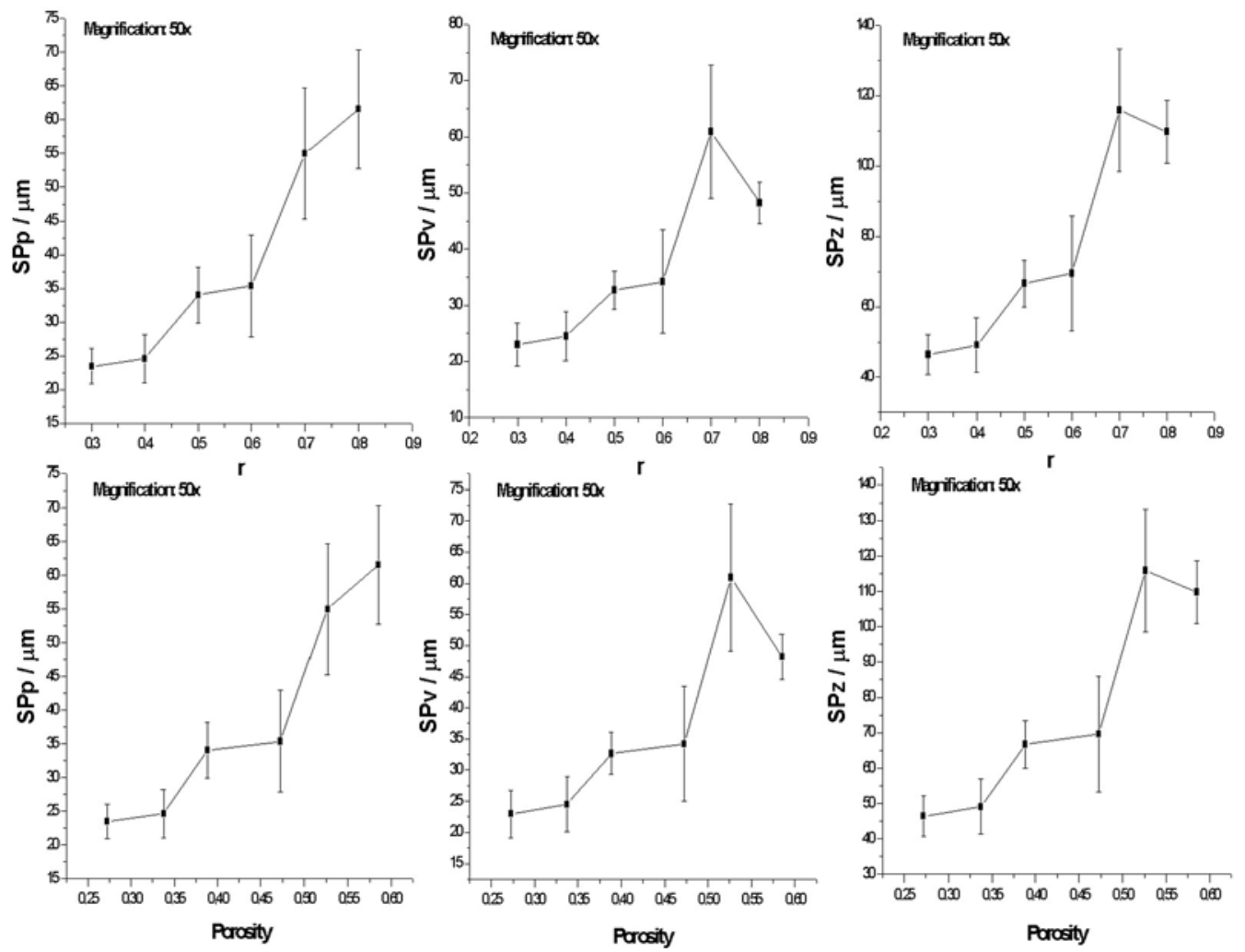

Fig. 5: 3D profile parameters $S P_{p}, S P_{v}, S P_{z}$ as dependents on the water-to-cement ratio $r$ and porosity $P$ - magnification $50 \times$
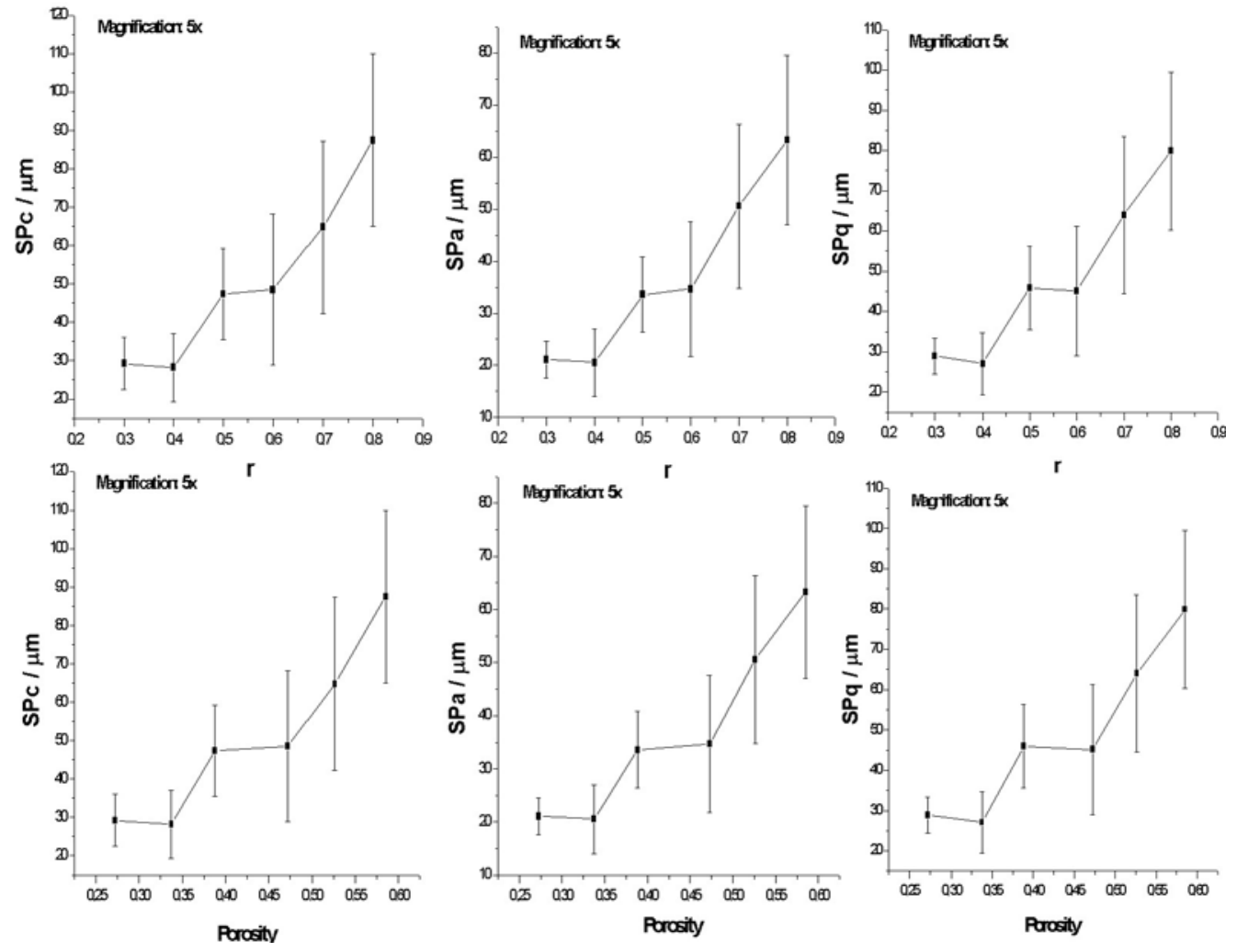

Fig. 6: 3D profile parameters $S P_{c}, S P_{a}, S P_{q}$ as dependents on the water-to-cement ratio $r$ and porosity $P$ - magnification $5 \times$ 

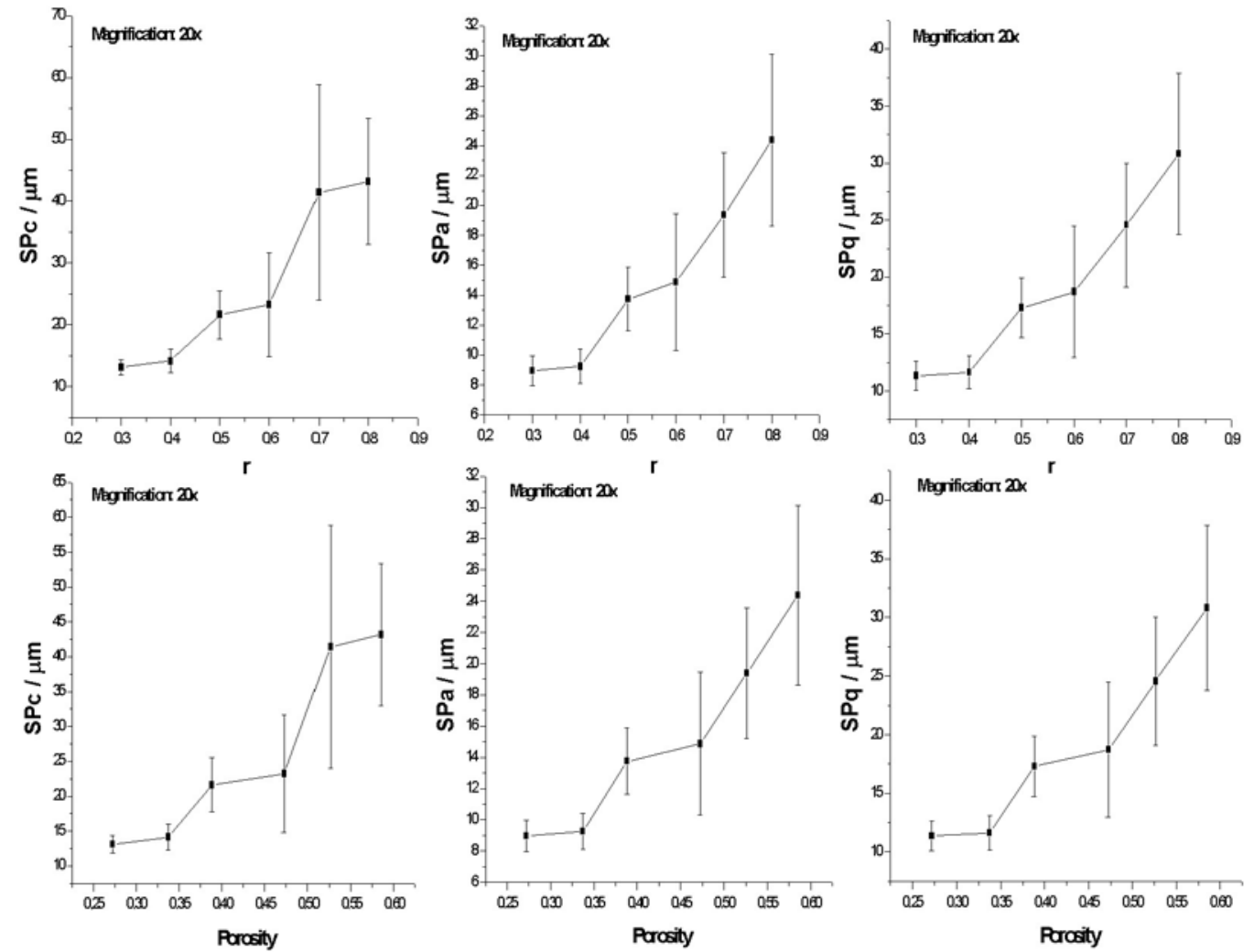

Fig. 7: 3D profile parameters $S P_{c}, S P_{a}, S P_{q}$ as dependents on the water-to-cement ratio $r$ and porosity $P$ - magnification $20 \times$
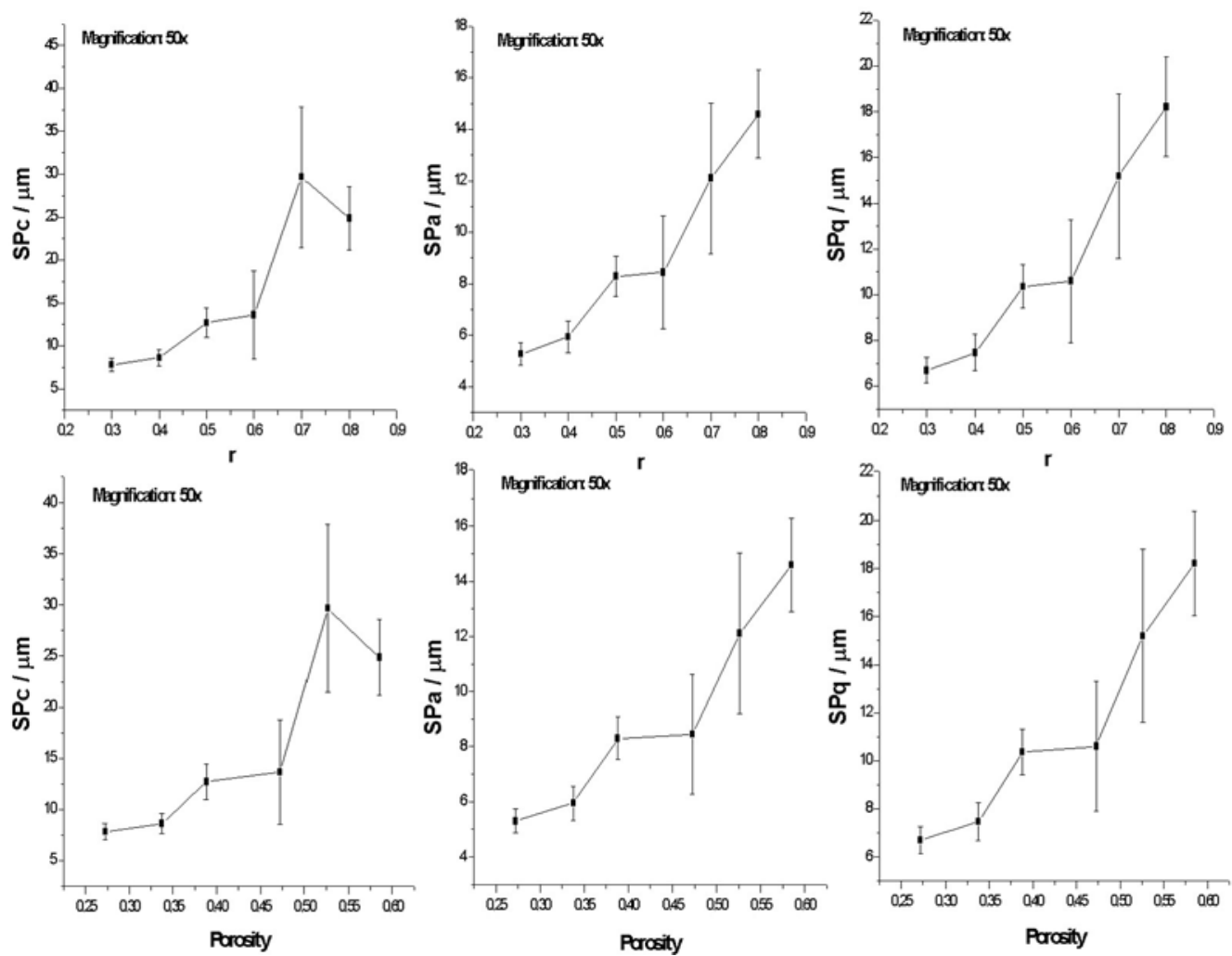

Fig. 8: 3D profile parameters $S P_{c}, S P_{a}, S P_{q}$ as dependents on the water-to-cement ratio $r$ and porosity $P$ - magnification $50 \times$ 

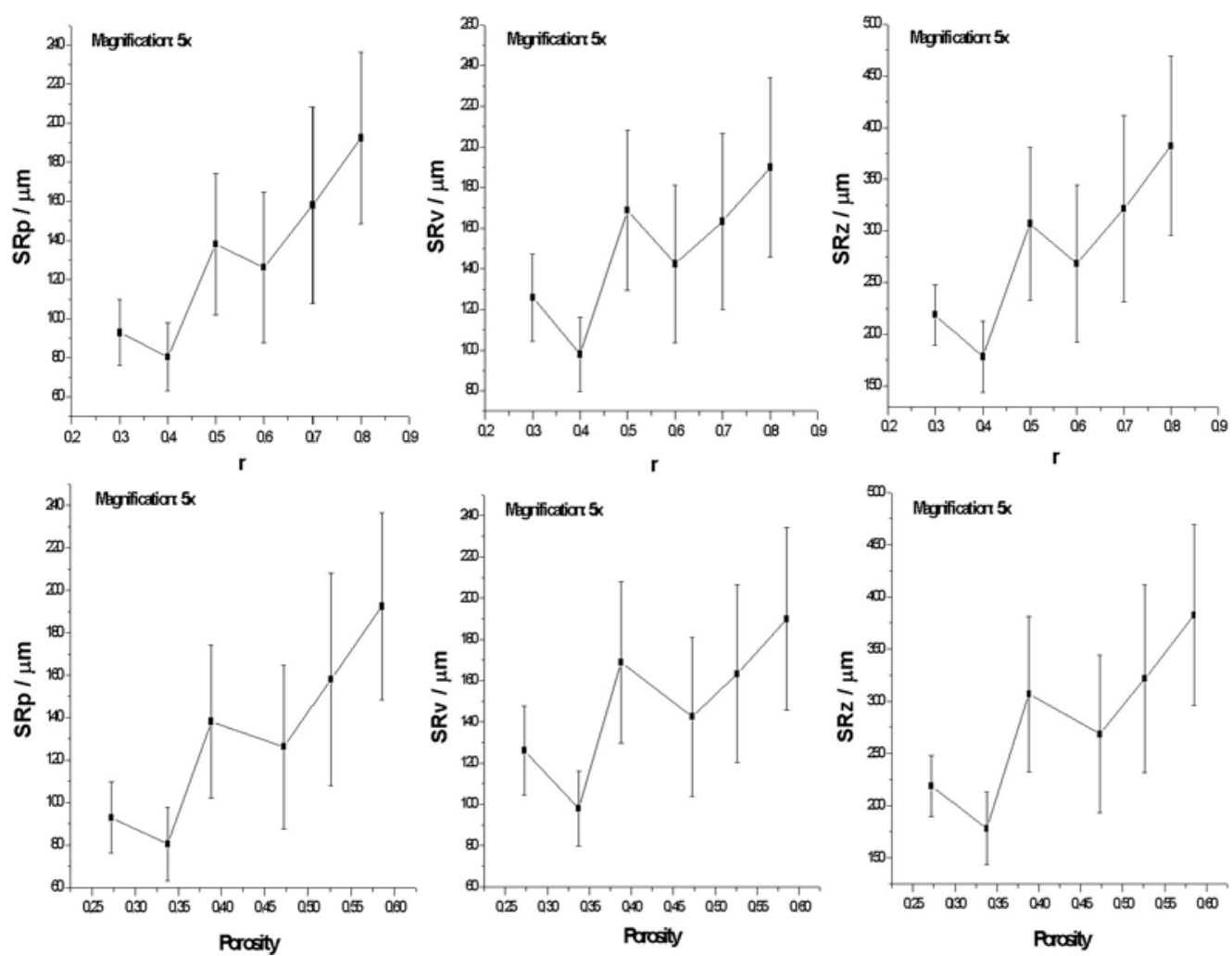

Fig. 9: 3D roughness parameters $S R_{p}, S R_{v}, S R_{z}$ as dependents on the water-to-cement ratio $r$ and porosity $P$ - magnification $5 \times$

face irregularities is clearly visible, not only with $S P_{z}$ but also with $S P_{c}$. Parameter $S P_{c}$ actually represents $S P_{z}$ averaged over the whole fracture surface, and in this sense they are mutually related.

Finally, noting that the shapes of the $S P$ and $S R$ graphs are similar (compare, e.g. Figures 6 and 12), it is also noted that their characteristic length scales do not differ enough to modify their vertical arrangements.

\subsection{Dependences on porosity}

The lower halves of Figures 3-15 show the graphs of the dependences of the profile parameters $S P_{p}(P)$, $S P_{v}(P), S P_{z}(P), S P_{c}(P), S P_{a}(P), S P_{q}(P)$ and the roughness parameters $S R_{p}(P), S R_{v}(P), S R_{z}(P)$, $S R_{c}(P), S R_{a}(P), S R_{q}(P), S R_{z j i s}(P)$ on porosity $P$. All of the above discussion on the water-to-cement ratio $r$ in Section 4.1 can be applied to the dependences on porosity $P$. This is because there is a strong correlation between these two quantities, as is also illustrated in Figure 2. The unambiguous and almost linear correspondence between $P$ and $r$ of the studied specimens in the interval $r \in(0.3,0.8)$ ensures an unambiguous, almost linear transition between the $r$-axes and the $P$-axes of the graphs in Figures $3-15$. This in turn guarantees almost identical shapes of the graphs, regardless whether they are based on $r$ variables or on $P$-variables.
The same strong dependences of the surface irregularity parameters $S P / S R$ on $r$ - or $P$-quantities together with their identical graphical shapes are convincing evidence of the governing roles of $r$ and $P$ related to the irregularity of the fracture surfaces of highly porous hydrated cement pastes. The influence of porosity on surface irregularity is not necessarily only a specific feature of porous cement pastes, but may be an inherent feature of other porous solid materials.

The finding that surface irregularity is prevalently determined by porosity is in accordance with the observation of Ponson and others $[10,11]$. They studied the roughness of the fracture surfaces of glass ceramics made of small glass beads sintered in bulk with porosity that could be varied within a certain interval up to $\sim 30 \%$. They observed that the twodimensional profile parameter [10] increased in value with increasing porosity.

Porosity seems to be a major factor governing the height irregularities of the fracture surfaces of porous solids. The roughness of the fracture remnants may be inferred from the porosity values, and conversely the porosity may be assessed from the surface roughness of the fracture remnants. Unfortunately, there is no exact theory to support this close relation between porosity and surface irregularity. This task remains as a challenge for future research. 

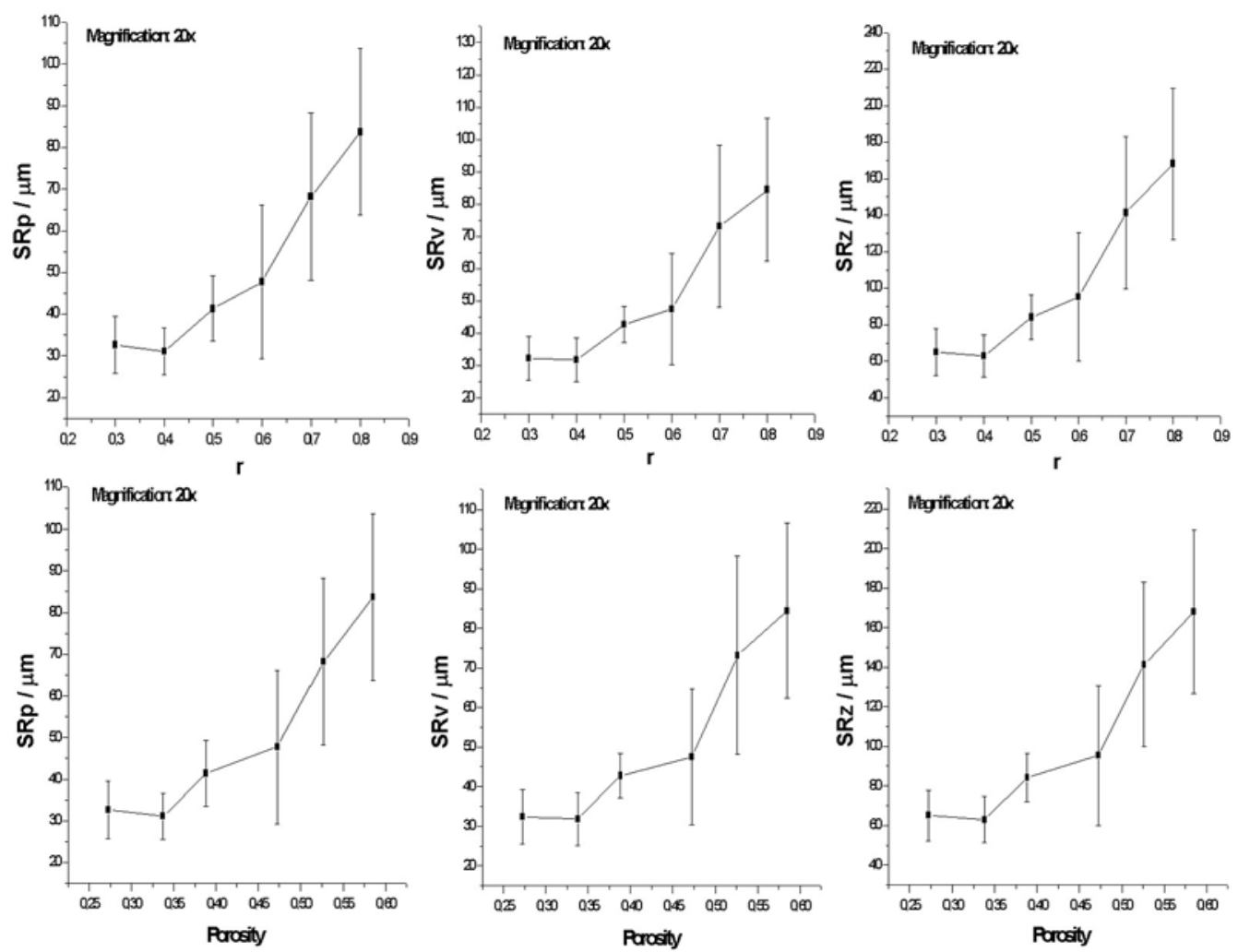

Fig. 10: 3D roughness parameters $S R_{p}, S R_{v}, S R_{z}$ as dependents on the water-to-cement ratio $r$ and porosity $P$ - magnification $20 \times$
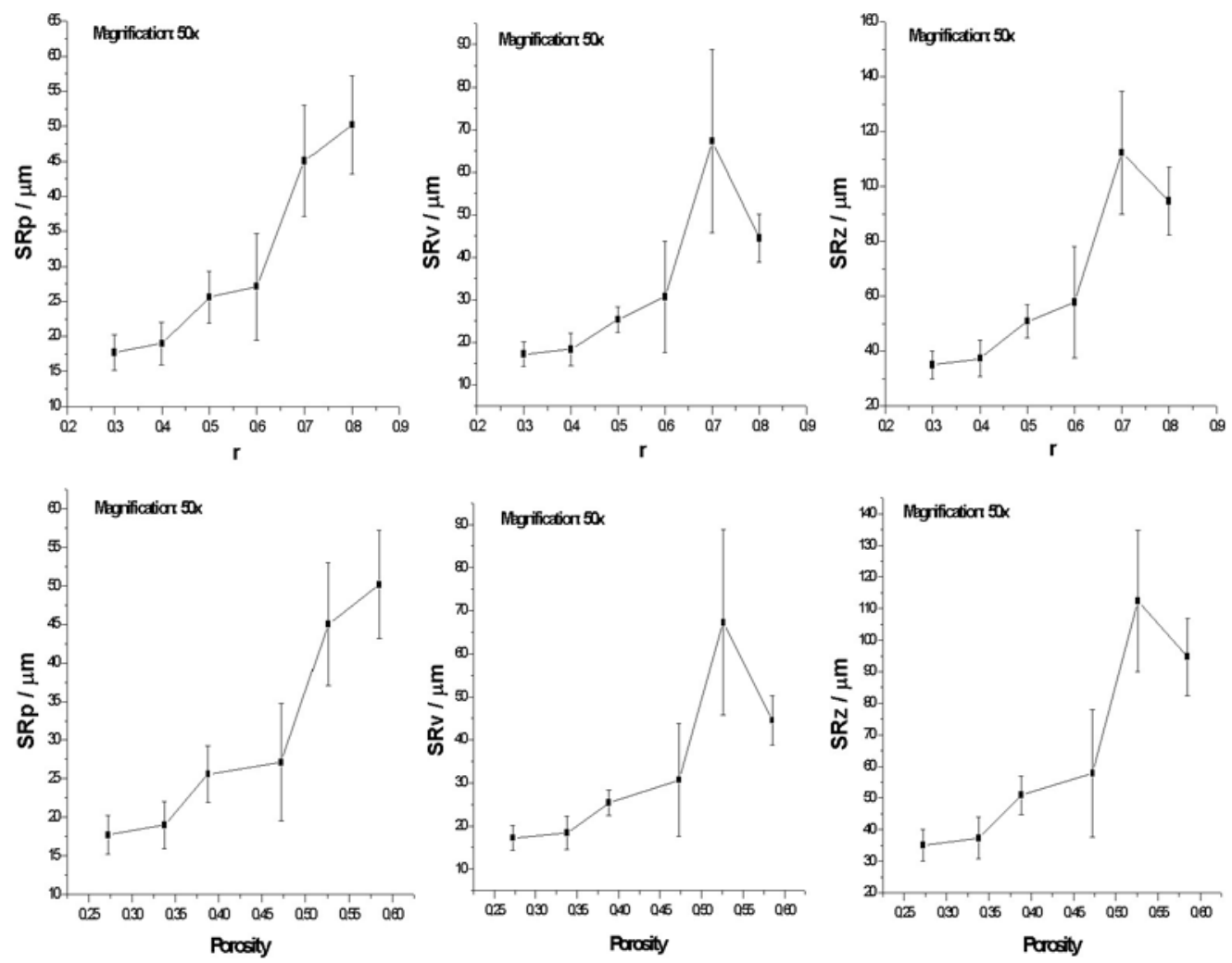

Fig. 11: 3D roughness parameters $S R_{p}, S R_{v}, S R_{z}$ as dependents on the water-to-cement ratio $r$ and porosity $P$ - magnification $50 \times$ 

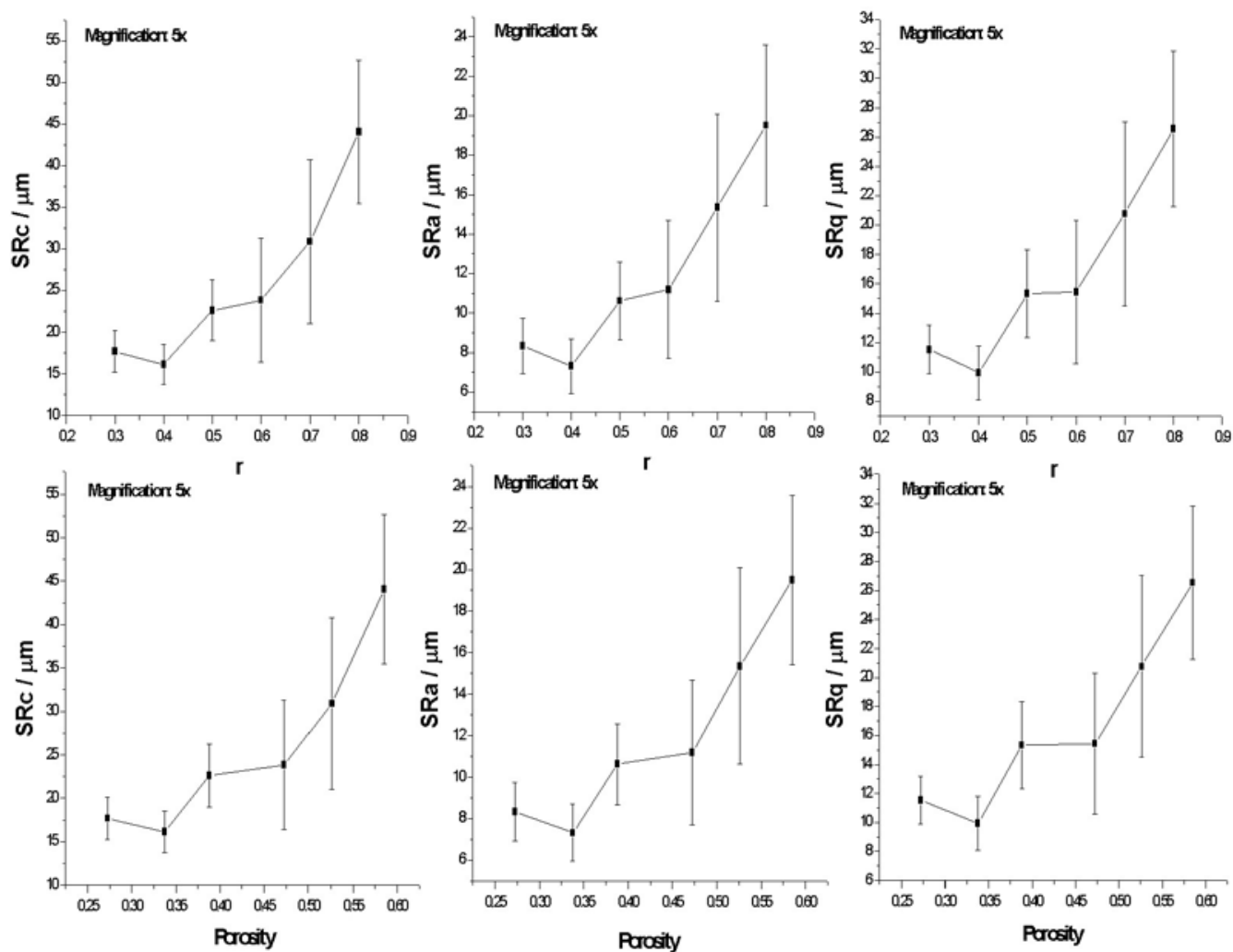

Fig. 12: 3D roughness parameters $S R_{c}, S R_{a}, S R_{q}$ as dependents on the water-to-cement ratio $r$ and porosity $P$ - magnification $5 \times$
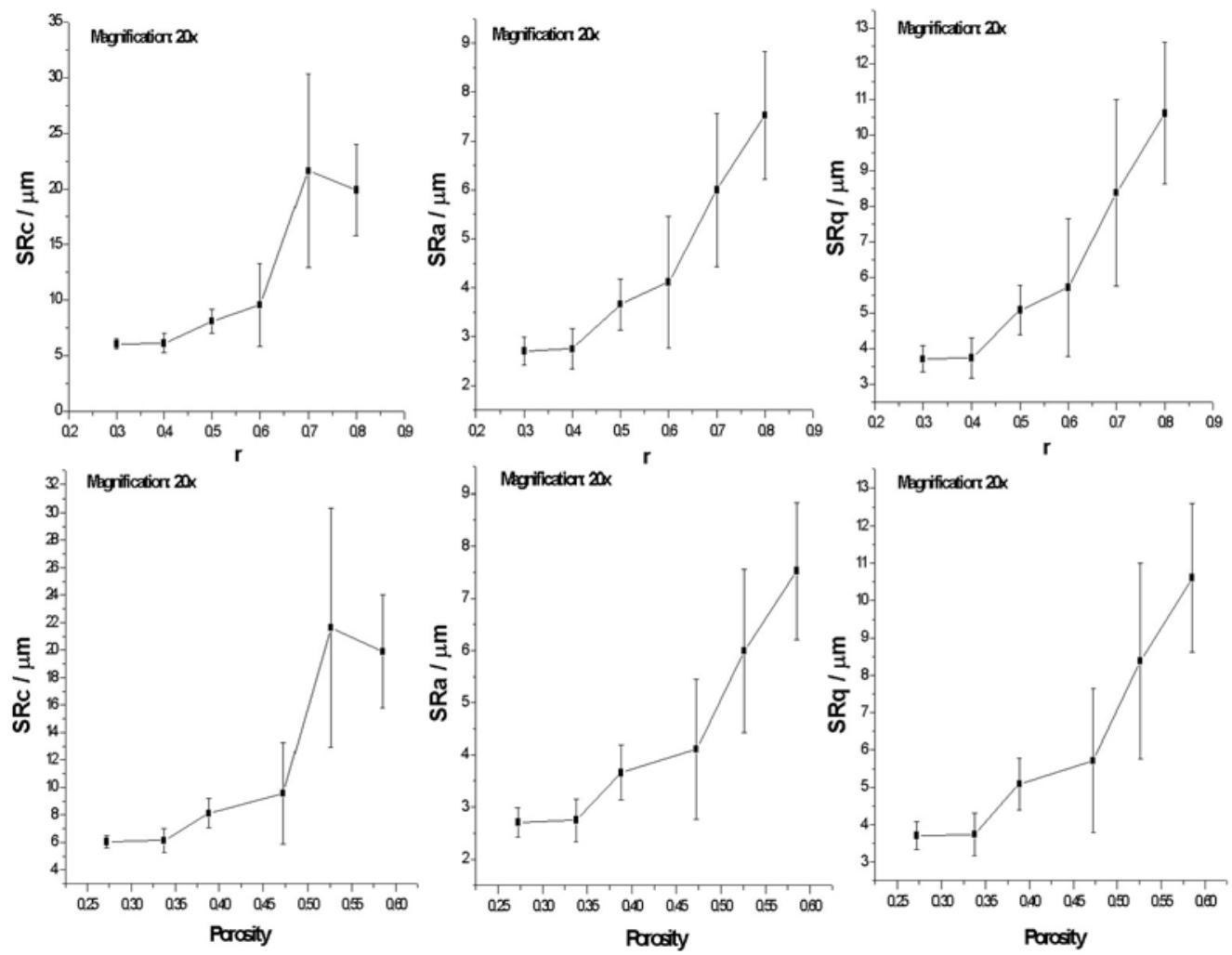

Fig. 13: 3D roughness parameters $S R_{c}, S R_{a}, S R_{q}$ as dependents on the water-to-cement ratio $r$ and porosity $P$ - magnification $20 \times$ 

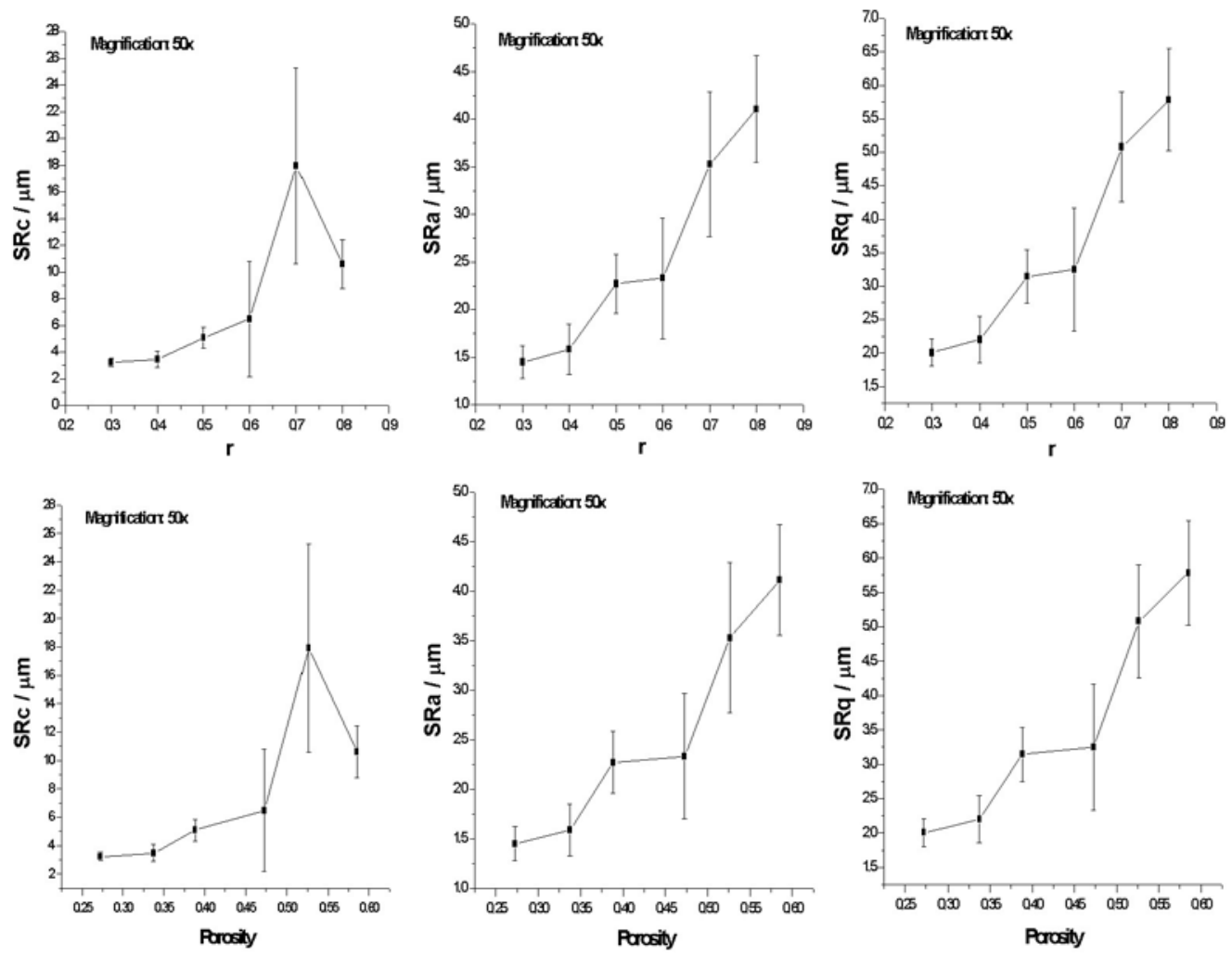

Fig. 14: 3D roughness parameters $S R_{c}, S R_{a}, S R_{q}$ as dependents on the water-to-cement ratio $r$ and porosity $P$ - magnification $50 \times$
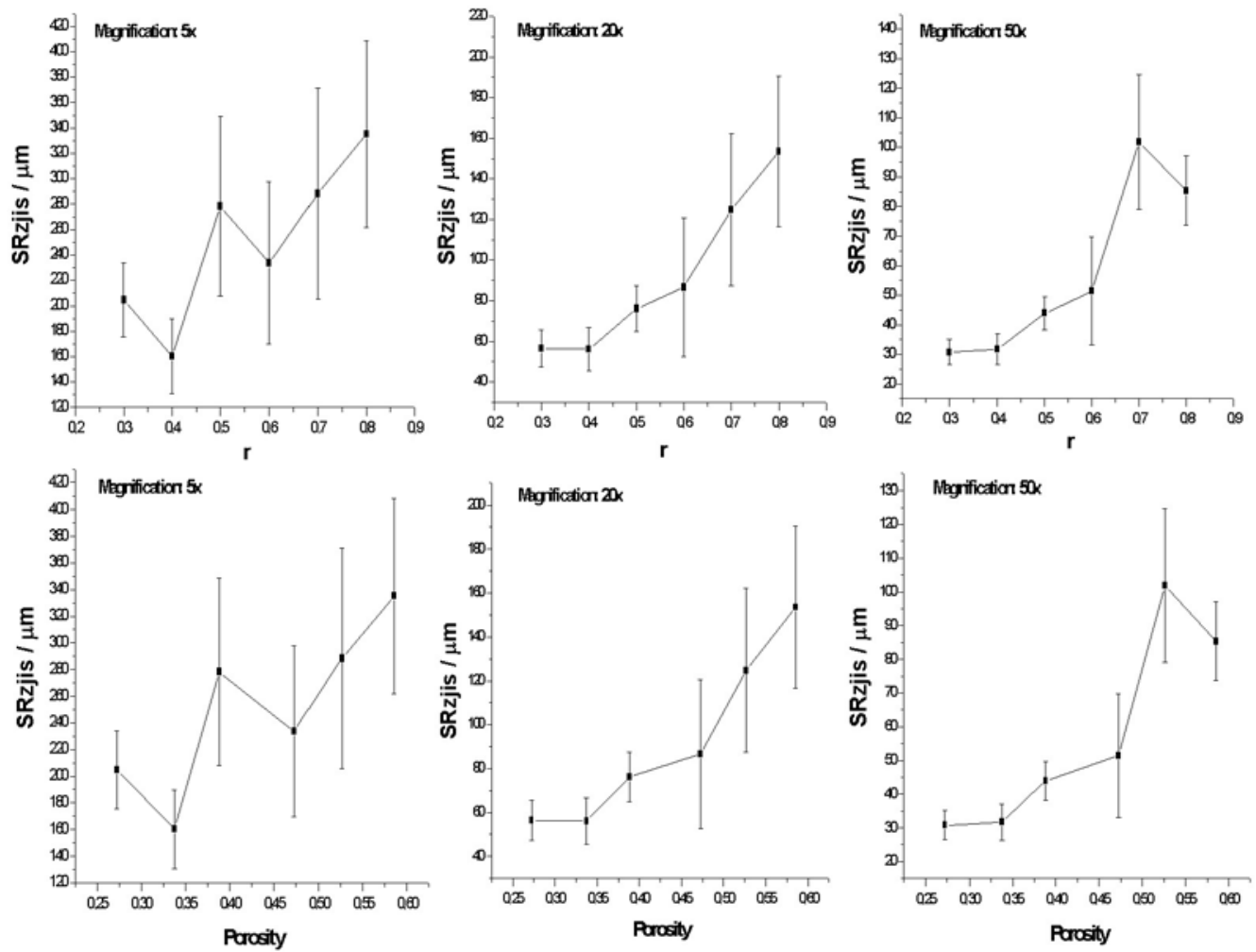

Fig. 15: 3D roughness parameter $S R_{z j i s}$ as dependent on the water-to-cement ratio $r$ and porosity $P$ - magnifications $5 \times, 20 \times$ and $50 \times$ 


\section{Conclusion}

An extensive study of the fracture surfaces of hydrated cement pastes has been performed using 3D $S P$ profile parameters and $S R$ roughness parameters. Thirteen 3D parameters of different kinds have been employed to describe and analyze surface irregularities on 106 specimens of cement pastes prepared with 6 different water-to-cement ratios. Each fracture surface has been tested on 5 different sites, so that each value of the $3 \mathrm{D}$ surface parameters belonging to the particular ratio $r$ has been averaged over 80 measured values. This essential statistical relevancy has been associated with each experimental point on the plotted graphs. This has enabled us to specify some of our preliminary results [9] more precisely and reliably.

The microscopic measurements were performed in triplicate in three different magnifications $5 \times, 20 \times$ and $50 \times$, resulting in 78 graphs describing the behavior of the surface irregularities of fractured specimens in dependence on various water-to-cement ratios and porosities.

The 3D $S P$ profile parameters and $S R$ roughness parameters have proved to be capable of analyzing the geometric irregularities of the surfaces of hydrated cement pastes and of providing information on height differences, on morphological singularities and on some missing surface features, e.g. suppressed protrusions (peaks) or depressions (valleys).

The results achieved in different magnifications have shown that the values of $3 \mathrm{D}$ surface parameters $S P / S R$ are dependent on the length scales, and for this reason their values are reduced when using a larger magnification and their values are expanded at small magnifications.

It has been shown that $S P_{a}, S P_{q}$ are the most reliable of all the studied parameters as regards the minimum statistical scatter of the processed values. The specific distribution of the length scales of the studied fracture surfaces of cement pastes has proved to be well treated in magnification $20 \times$, at which the $3 \mathrm{D}$ profile parameters $S P$ and roughness parameters $S R$ provide the most stable values. Naturally, this does not mean at all that the fracture surfaces of other materials with differently distributed length scales of surface irregularities will also prefer magnification $20 \times$.

The present study has shown a close relation between surface irregularities and the porosity of hydrated cement pastes. Since porosity is influenced by the water-to-cement ratio, a close relation has also been found between the surface irregularities and the water-to-cement ratio. For this reason, the surface irregularities, quantified by parameters $S P / S R$, show very similar analytical dependences both on porosity $P$ and on water-to-cement ratio $r$.
The initial value of the water-to-cement ratio used for mixing cement paste is one of the main factors that decides about the future porosity of cement hydrates, and is also influential for the surface irregularities of the fracture remnants of this material.

\section{Acknowledgement}

This work was supported by the Ministry of the Czech Republic under Contract no. ME 09046 (Kontakt).

\section{References}

[1] Lange, D. A., Jennings, H. M., Shah, S. P.: A fractal approach to understanding cement paste microstructure, Ceram. Trans. 16 (1992) 347-363.

[2] Issa, M. A., Hammad, A. M.: Fractal characterization of fracture surfaces in mortar, Cem. Concr. Res. 23 (1993) 7-12.

[3] Lange, D. A., Jennings, H. M., Shah, S. P.: Analysis of surface-roughness using confocal microscopy, J. Mater. Sci. 28 (14) (1993) $3879-3884$.

[4] Lange, D. A., Jennings, H. M., Shah, S. P.: Relationship between fracture surface roughness and fracture behavior of cement paste and mortar, J. Am. Ceram. Soc. 76 (3) (1993) 589-597.

[5] Zampini, D., Jennings, H. M., Shah, S. P.: Characterization of the paste-aggregate interfacial transition zone surface-roughness and its relationship to the fracture-toughness of concrete, J. Mater. Sci. 30 (12) (1995) 3149-3154.

[6] Lange, D. A., Quyang, C., Shah, S. P.: Behavior of cement-based matrices reinforced by randomly dispersed microfibers, Adv. Cem. Bas. Mater. 3 (1) (1996) 20-30.

[7] Abell, A. B., Lange, D. A.: Fracture mechanics modeling using images of fracture surfaces, 35 (31-32) (1997) 4025-4034.

[8] Nichols, A. B., Lange, D. A.: 3D surface image analysis for fracture modeling of cementbased materials, Cem. Conc. Res. 36 (2006) 1 098-1 107.

[9] Ficker, T., Martišek, D., Jennings, H. M.: Roughness of fracture surfaces and compressive strength of hydrated cement pastes, Cem. Conr. Res. 40 (2010) 947-955.

[10] Ponson, L.: Crack propagation in disordered materials; How to decipher fracture surfaces (Ph.D. Thesis, Univ. Paris., 2006) (http://pastel.paristech.org/2920/?). 
[11] Ponson, L., Auradou, H., Pessel, M., Lazarus, V., Hulin, J. P.: Failure mechanisms and surface roughness statistics of fractured Fontainebleau sandstone, Phys. Rev. E $\mathbf{7 6}$ (2007) 036108/1-036108/7.

Prof. RNDr. Tomáš Ficker, DrSc.

Phone: +420 541147661

E-mail: ficker.t@fce.vutbr.cz

Department of Physics

Faculty of Civil Engineering

Brno University of Technology

Veveří 95, 66237 Brno, Czech Republic
Assoc. Prof. PaedDr. Dalibor Martišek, Ph.D.

Department of Mathematics

Faculty of Mechanical Engineering

Brno University of Technology

Technická 2896/2, 61600 Brno, Czech Republic

Adjunct Professor Hamlin M. Jennings, Ph.D.

Department of Civil and Environmental Engineering Massachusetts Institute of Technology

77 Massachusetts Avenue, Cambridge, MA, 02139, U.S.A. 